\title{
FATORES ASSOCIADOS AO USO DE MEDICAMENTOS POTENCIALMENTE INAPROPRIADOS EM IDOSOS URBANOS E RURAIS
}

\section{Eduarda Brum Guedes Salcher}

Enfermeira. Mestre em Envelhecimento Humano pela Universidade de Passo Fundo (UPF), Brasil.

\section{Marcos Paulo Dellani}

Enfermeiro, Mestre em Envelhecimento Humano, Docente do Instituto de Desenvolvimento Educacional do Alto Uruguai (IDEAU), Brasil.

\section{Marilene Rodrigues Portella}

Enfermeira. Docente do Programa de Pós-Graduação em Envelhecimento Humano da Universidade de Passo Fundo (UPF), Brasil.

\section{Marlene Doring}

Enfermeira. Docente do Programa de Pós-Graduação em Envelhecimento Humano da Universidade de Passo Fundo (UPF), Brasil.
Autor correspondente:

Eduarda Brum Guedes Salcher

E-mail: eduardabgs@hotmail.com
RESUMO: Este estudo objetivou verificar a associação entre uso de medicamentos potencialmente inapropriados e zona de moradia, condições de saúde, hábitos de vida e capacidade funcional de idosos urbanos e rurais. Trata-se de um estudo transversal, recorte de um estudo de base populacional sobre condições de vida e saúde de idosos residentes nas zonas urbana e rural do município de Estação (RS). Por meio de inquérito domiciliar, os 313 idosos entrevistados autorreferiram informações pessoais e familiares, condições de saúde e hábitos de vida e avaliação funcional. As informações coletadas foram codificadas e armazenadas em um banco de dados. Os dados foram analisados e as medicações utilizadas e sua classificação quanto aos Critérios de Beers, versão 2012 foram identificadas. O estudo evidenciou uma prevalência elevada quanto ao uso de medicamentos potencialmente inapropriados em idosos. As classes terapêuticas de medicamentos mais utilizadas foram drogas antiarrítmicas, benzodiazepínicos e anti-inflamatórios não esteroidais. Identificou-se associação positiva entre o uso de medicamentos potencialmente inapropriados e dor, quedas, atividade física, diabetes, problemas no sono, nervosismo, problemas cardíacos, depressão e atividades básicas da vida diária. Tal evidência reforça a necessidade de capacitação e sensibilização dos profissionais de saúde para a avaliação da terapia medicamentosa.

PALAVRAS-CHAVE: Medicamentos potencialmente inapropriados; Terapia farmacológica; Saúde da população rural; Polimedicaçao; Envelhecimento.

\section{FACTORS ASSOCIATED WITH THE USE OF MEDICINES POTENTIALLY INADEQUATE FOR URBAN AND RURAL ELDERLY PEOPLE}

\begin{abstract}
The association between the use of potentially inadequate medicines and living sites, health conditions, habits and functional capacity of urban and rural elderly people is analyzed. Current transversal study is a section of a population-based investigation on health and life conditions of elderly people living in the urban and rural zones of the municipality of Estação RS Brazil. A home survey was conducted with 313 elderly people who provided their personal and family data, health conditions, habits and functional evaluation. Information was codified and stored in a data base. Data on medicines used and their classification were analyzed according to Beer's Criteria v. 2012. Investigation revealed high prevalence of medicine which was potentially inadequate for the elderly. Antiarrhythmic, benzodiazepines and non -steroid anti-inflammatory drugs were the most employed drug therapeutic classes. A positive association between the use of potentially inadequate medicines and pain, falls, physical activity, diabetes, insomnia, nervousness, heart condition, depression and daily basic activities was
\end{abstract}


identified. Results reinforce the need for capacitation and sensitiveness of health professionals to assess medicinal therapy.

KEY WORDS: Potentially inadequate medicines; Pharmacological therapy; Health of the rural population; Polypharmacy ; Aging.

\section{INTRODUÇÃO}

O cenário atual de transformações sociais e demográficas é pautado por modificações nos padrões de vida da população, marcando um período de controle de doenças infectocontagiosas, tendo em vista o crescimento das doenças crônicas não transmissíveis, e suas repercussões para o período de transição epidemiológica vivido ${ }^{1-4}$.

As melhorias nas condições sociais e de saúde da população fizeram com que se atingisse maior longevidade. Com o incremento de uma parcela maior de idosos na sociedade, houve aumento das doenças crônico-degenerativas. Dados divulgados em pesquisas demonstram que $77,4 \%$ dos idosos brasileiros são portadores de, ao menos, uma doença crônica ${ }^{4-6}$. Logo, com o aumento de doenças, sobretudo as crônicas e limitantes, há necessidade de cuidados constantes em curto e longo prazo, bem como o uso contínuo de medicações que deem conta de suprir o tratamento das patologias ${ }^{7}$, manter a qualidade e a sobrevida ${ }^{8 \cdot 10}$.

Da mesma forma em que se faz necessário o uso de múltiplos medicamentos, para manutenção do regime terapêutico dos idosos, sabe-se também que pelas alterações farmacodinâmicas e farmacocinéticas, próprias do envelhecimento, o uso de medicamentos expõe o indivíduo a riscos. Sobretudo, pelo fato de grande parte dos idosos utilizarem diversas fórmulas farmacológicas, o que favorece interações medicamentosas e reações adver$\operatorname{sas}^{11-14}$.

Neste sentido, determinados fármacos, os medicamentos potencialmente inapropriados (MPI) são considerados impróprios, pois seus riscos superam os benefícios do fármaco, tendo chances elevadas de provocar efeitos colaterais e reações adversas a medicamentos em idosos, devendo ser evitados, independente da dose e do tratamento utilizado ${ }^{15,16}$. Desse modo, fazem-se ne- cessários mecanismos de avaliação para o uso racional de medicamentos. Uma das estratégias é o critério de Beers, o mesmo é utilizado na análise da terapêutica farmacológica do paciente idoso, com vistas a identificar os MPI, o qual apresenta uma lista de 53 fármacos considerados inapropriados, tendo sustentação de cunho científico ${ }^{17-19}$. $\mathrm{O}$ presente estudo objetivou verificar a associação entre uso de medicamentos potencialmente inapropriados $\mathrm{e}$ zona de moradia, condições de saúde, hábitos de vida e capacidade funcional.

\section{METODOLOGIA}

Estudo transversal de base populacional, recorte da pesquisa realizada no Programa de Pós-graduação em Envelhecimento Humano sobre as condições de vida e saúde de idosos residentes nas zonas urbana e rural do município de Estação (RS).

A população do estudo foi composta por indivíduos residentes no município, em meios urbano e rural, com idade igual ou superior a 60 anos. Foi utilizado como fonte de informação populacional o sistema da Secretaria de Saúde do município de Estação, a qual informou que havia 992 idosos residentes em 12 microáreas de saúde no município. Destas, dez localizadas na zona urbana, com um total de 837 idosos; duas, na zona rural, com 155 idosos. Do total dessa população, representada em $57 \%$ pelo sexo feminino e $43 \%$ pelo sexo masculino, $84 \%$ viviam na zona urbana e $16 \%$ na zona rural. Para o cálculo amostral o erro aceitável adotado foi de 0,05 e intervalo de confiança de $95 \%$ resultando em 382 idosos. Considerando possíveis perdas de $10 \%$ (não elegibilidade, recusas, entre outras), um número adicional de idosos foi incluído como margem de segurança, totalizando 420 idosos selecionados. Para este estudo foi considerada a proporção de idosos por zona, mantendo o percentual de $57 \%$ para o sexo feminino e $43 \%$ para o sexo masculino. Dos 277 idosos da zona urbana, 158 são do sexo feminino e 119 do sexo masculino; na zona rural, dos 67 idosos, 38 são do sexo feminino e 29 do sexo masculino. Os idosos foram selecionados, aleatoriamente, com base nos registros da Estratégia de Saúde da Família. Inicialmente, foram listados por zonas de residência e sexo e, 
a seguir, foram selecionados por amostragem aleatória, mantendo as proporções estipuladas pela amostra.

Foram considerados como critérios de inclusão: ter idade igual ou superior a 60 anos; residir há pelo menos seis meses no município; possuir, no ato da entrevista, condições cognitivas para responder ao questionário e/ou contar com a presença de um familiar ou cuidador para auxiliar ou efetuar as respostas. Consideraram-se como perdas os indivíduos que não foram localizados após três tentativas, em horários diferentes, e constatar com a vizinhança sua ausência; mudança de residência para outro município; por óbito no período da coleta; indivíduos elegíveis que se recusaram a participar. Foram excluídos os idosos com déficit auditivo ou visual que não contassem com um cuidador ou responsável para auxiliar na entrevista.

Coletaram-se os dados por meio de inquérito domiciliar, utilizando-se um questionário estruturado balizado no instrumento empregado pela Pesquisa Saúde, Bem-Estar e Envelhecimento-SABE. Consideraram-se as seções: A) Informações pessoais e familiares, D) Condições de saúde e hábitos de vida e seção E) Avaliação funcional. As informações coletadas foram codificadas e armazenadas em um banco de dados. Para este estudo, no referido banco observaram-se as indicações das medicações autorreferidas e na classificação dos MPI conforme os Critérios de Beers, versão 2012, que contêm os princípios ativos dos fármacos considerados inapropriados. Essa classificação apresenta oito tópicos de análise intitulados categorias terapêuticas ou droga. Sendo as categorias: anticolinérgicos; antitrombóticos; anti-infecciosos; cardiovasculares; sistema nervoso central; endócrinos; gastrintestinal e dor. A partir das medicações informadas/ autorreferidas, foram identificados os idosos que faziam uso de medicamentos para compor a população final de estudo, totalizando um número de 313 idosos.

A análise descritiva inclui cálculos de proporções e intervalos de confiança de $95 \%$ para as variáveis categóricas. Nas variáveis numéricas foi calculada a média, mediana e desvio-padrão. Foram realizadas análises bivariadas entre o desfecho MPI e as variáveis independentes (sexo, idade, hábitos de vida, condições de saúde, condições sociodemográficas, local de moradia, capacidade funcional). Para associação entre variáveis categóricas dicotômicas foi utilizado o teste qui-quadrado. A pesquisa foi aprovada pelo Comitê de Ética em Pesquisa da Universidade de Passo Fundo, sob o parecer n $n^{\circ}$ 017/2011. A coleta de dados seguiu as diretrizes preconizadas pela resolução 466/12 do Conselho Nacional de Saúde.

\section{RESULTADOS}

Dos 313 idosos incluídos no estudo, 49,2\% utilizavam algum medicamento potencialmente inapropriado. Destes, $60,4 \%$ eram do sexo feminino. Houve predomínio de participantes na faixa etária entre 60 a 79 anos de idade, com o total de $85,3 \%$, dos quais $14,7 \%$ tinham mais de 80 anos. A grande maioria $(87,2 \%)$ era de pele branca. Destaca-se, quanto ao estado marital, que $73,0 \%$ dos idosos entrevistados eram casados, que $49,4 \%$ viviam com somente um familiar na residência, dos quais 50,6\% vivem com renda familiar entre três e cinco salários. Quanto à zona de moradia, 84,3\% eram moradores da área urbana. Idosos que sabiam ler e escrever corresponderam a $85,3 \%$ dos entrevistados. Referente ao uso de medicamentos, $67,3 \%$ dos entrevistados utilizava de um1 a quatro medicamentos, já 32,3\% dos idosos faziam uso de cinco ou mais medicamentos concomitantemente, caracterizando o uso de polifarmácia.

Quanto à cognição, observou-se que 83,1\% dos idosos mantinham a cognição preservada e 7,3\% possuíam dependência para atividades básicas da vida diária (ABVD). No que se refere à prática de atividades físicas, $51,4 \%$ dos entrevistados praticava algum tipo de atividade. Referiram dificuldade visual $83,3 \%$ dos participantes e 60,4\% referiram sentir dor contínua há mais de três meses. A hipertensão foi a patologia indicada por $82,1 \%$ dos idosos.

Na Tabela 1 é apresentada a distribuição dos grupos de MPI mais utilizados pelos idosos participantes do estudo. 
Tabela 1. Distribuição do uso de MPI conforme o Critério de Beers. Estação, 2011

\begin{tabular}{ccc}
\hline GRUPO MPI & $\mathrm{n}$ & $\%$ \\
\hline Cardiovascular (n=53-34,9\%) & & \\
Bloqueadores Alfa & 7 & 13,2 \\
Alfa-Agonistas centrais & 3 & 5,6 \\
Drogas antiarrítmicas & 32 & 60,4 \\
Digoxina & 5 & 9,4 \\
Espironolactona & 6 & 11,3 \\
\hline
\end{tabular}

Sistema Nervoso Central $(n=10-6,6 \%)$

\begin{tabular}{lcc} 
ADTs terciários & 10 & 14,7 \\
Antipsicóticos & 1 & 1,5 \\
Tioridazina & 3 & 4,4 \\
Barbituratos & 2 & 2,9 \\
$\quad$ Benzodiazepínicos & 52 & 76,4 \\
\hline Endócrino (n=18-11,8\%) & & \\
$\quad$ Andrógenos & 6 & 33,4 \\
Estrogênios & 1 & 5,5 \\
Insulina & 10 & 55,6 \\
$\quad$ Megestrol & 1 & 5,5 \\
\hline Dor (n=71-46,7\%) & & \\
AINES & 68 & 95,7 \\
$\quad$ Relaxantes Musculares & 3 & 4,2 \\
\hline
\end{tabular}

Foram encontrados MPI que correspondem aos grupos cardiovasculares, sistema nervoso central, endócrino e dor. Drogas antiarrítmicas com 60,4\%, benzodiazepínicos com 76,4\% e anti-inflamatórios não esteroidais (AINES) 95,7\% foram as medicações mais utilizadas nos referidos grupos.

Na Tabela 2 é apresentada a análise bivariada com a associação entre o uso de MPI com as variáveis sociodemográficas.
Tabela 2. Resultados da análise bivariada entre uso de MPI e variáveis sociodemográficas. Estação, RS, 2011

\begin{tabular}{|c|c|c|c|c|}
\hline \multirow[b]{2}{*}{ Variáveis } & \multicolumn{3}{|c|}{ MPI } & \multirow[b]{2}{*}{$p$} \\
\hline & Total & Sim n (\%) & Não n (\%) & \\
\hline Sexo & & & & 0,37 \\
\hline Masculino & 124 & $52(41,9)$ & $72(58,1)$ & \\
\hline Feminino & 189 & $102(54,0)$ & $87(46,0)$ & \\
\hline Faixa Etária & & & & 0,17 \\
\hline $0-79$ & 266 & $123(46,2)$ & $143(53,8)$ & \\
\hline 80 ou mais & 46 & $30(65,2)$ & $16(34,8)$ & \\
\hline Ler e Escrever & & & & 0,056 \\
\hline Sim & 267 & $125(46,8)$ & $142(53,2)$ & \\
\hline Não & 45 & $28(62,2)$ & $17(37,8)$ & \\
\hline Estado marital & & & & 0,001 \\
\hline Casado & 227 & $99(43,6)$ & $128(56,4)$ & \\
\hline Não casado & 84 & $54(64,3)$ & $30(35,7)$ & \\
\hline Renda Familiar & & & & 0,020 \\
\hline Até 1 salário & 20 & $15(75,0)$ & $5(25,0)$ & \\
\hline De 1 a 2 sal. & 99 & $49(49,5)$ & $50(50,5)$ & \\
\hline De 3 a 5 sal. & 158 & $79(50,0)$ & $79(50,0)$ & \\
\hline $\begin{array}{r}\text { Acima de } 5 \\
\text { sal. }\end{array}$ & 35 & $11(31,4)$ & $24(68,6)$ & \\
\hline Percepção saúde & & & & 0,001 \\
\hline Muito boa/boa & 156 & $61(39,1)$ & $95(60,9)$ & \\
\hline $\begin{array}{r}\text { Muito ruim/ } \\
\text { ruim }\end{array}$ & 155 & $91(58,7)$ & $64(41,3)$ & \\
\hline Cor da pele & & & & 0,252 \\
\hline Branco & 272 & $130(47,8)$ & $142(52,2)$ & \\
\hline Não branco & 40 & $23(57,5)$ & $17(42,5)$ & \\
\hline Zona de moradia & & & & 0,061 \\
\hline Urbano & 205 & $93(45,4)$ & $112(54,6)$ & \\
\hline Rural & 108 & $61(56,5)$ & $47(43,5)$ & \\
\hline Reside & & & & 0,087 \\
\hline Sozinho & 46 & $28(60,9)$ & $18(39,1)$ & \\
\hline Acompanhado & 267 & $126(47,2)$ & $141(52,8)$ & \\
\hline
\end{tabular}

$\mathrm{Na}$ análise bivariada, ocorreu associação significativa entre uso de MPI e as variáveis estado marital e autopercepção de saúde $(p<0,05)$, na variável saber ler e escrever houve associação limítrofe $(p=0,056)$. Conforme o demonstrado, idosos que percebem sua saúde como boa ou muito boa, fazem menor uso de MPI, e, proporcionalmente, os que classificam sua saúde como ruim ou muito ruim fazem maior uso de MPI.

$\mathrm{Na}$ análise entre MPI e as variáveis sexo, faixa etária, cor da pele, zona de moradia e residir sozinho ou acompanhado não foram encontradas associações estatis- 
ticamente significativas.

Na Tabela 3 estão apresentados os dados da análise bivariada entre o uso de MPI com as variáveis condições de saúde.

Tabela 3. Resultados da análise bivariada entre uso de MPI e variáveis condições de saúde. Estação, RS, 2011

\begin{tabular}{|c|c|c|c|c|}
\hline \multicolumn{5}{|c|}{ (Continua) } \\
\hline \multicolumn{5}{|c|}{ MPI } \\
\hline Variáveis & Total & $\operatorname{Sim} n(\%)$ & Não n (\%) & $p$ \\
\hline Dor & & & & 0,021 \\
\hline Sim & 189 & $102(54,0)$ & $87(46,0)$ & \\
\hline Não & 120 & $49(40,8)$ & $72(59,2)$ & \\
\hline Quedas & & & & 0,001 \\
\hline Sim & 79 & $51(64,6)$ & $28(35,4)$ & \\
\hline Não & 233 & $102(43,8)$ & $131(56,2)$ & \\
\hline Dificuldade visual & & & & 0,095 \\
\hline $\operatorname{Sim}$ & 260 & $133(51,2)$ & $127(48,8)$ & \\
\hline Não & 52 & $20(38,5)$ & $32(61,5)$ & \\
\hline
\end{tabular}

\begin{tabular}{lcrrr}
\hline Tabagismo & & & & 0,164 \\
Nunca fumou & 221 & $116(52,5)$ & $105(47,5)$ & \\
Ex-fumante & 66 & $27(40,9)$ & $39(59,1)$ & \\
Fuma & 25 & $10(40,0)$ & $15(60,0)$ &
\end{tabular}

\begin{tabular}{lcrrr}
\hline Uso de álcool & & & & 0,001 \\
Não usa & 225 & $126(56,0)$ & $99(44,0)$ & \\
1-3 dias semana & 33 & $10(30,3)$ & $23(69,7)$ & \\
4 dias ou mais & 55 & $18(32,7)$ & $37(67,3)$ & \\
\hline Atividade física & & & & 0,000 \\
Sim & 161 & $58(36,0)$ & $103(64,0)$ & \\
Não & 152 & $96(63,2)$ & $56(36,8)$ & \\
\hline Hipertensão & & & & 0,451 \\
Sim & 257 & $129(50,2)$ & $128(49,8)$ & \\
Não & 56 & $25(44,6)$ & $31(55,4)$ & \\
\hline Diabetes & & & & 0,014 \\
Sim & 49 & $32(65,3)$ & $17(34,7)$ & \\
Não & 264 & $122(46,2)$ & $142(53,8)$ & \\
\hline Obesidade & & & & 0,028 \\
Sim & 19 & $14(73,7)$ & $5(26,3)$ & \\
Não & 294 & $140(47,6)$ & $154(52,4)$ & \\
\hline Problemas sono & & & & 0 \\
Sim & 66 & $57(86,4)$ & $9(13,6)$ & \\
Não & 247 & $97(39,3)$ & $150(60,7)$ & \\
\hline Nervosismo & & & & 0,000 \\
Sim & 50 & $39(78,0)$ & $11(22,0)$ & \\
Não & 263 & $115(43,7)$ & $148(56,3)$ & \\
\hline
\end{tabular}

(Conclusão)

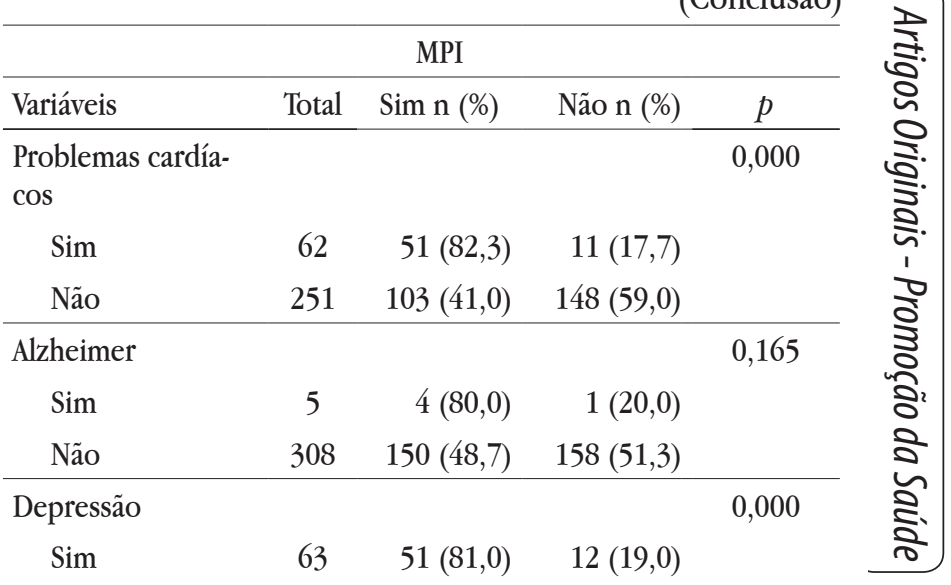

\begin{tabular}{lrrrr}
$\quad$ Não & 250 & $103(41,2)$ & $147(58,8)$ & \\
\hline Osteoporose & & & & 0,013 \\
$\quad$ Sim & 40 & $27(67,5)$ & $13(32,5)$ & \\
Não & 273 & $127(46,5)$ & $146(53,5)$ & \\
\hline Problemas circula- & & & & 0,000
\end{tabular}
tórios

\begin{tabular}{lcrrr} 
Sim & 96 & $79(82,3)$ & $17(17,7)$ & \\
$\quad$ Não & 217 & $75(34,6)$ & $142(65,4)$ & \\
\hline Problemas coluna & & & & 0,132 \\
$\quad$ Sim & 115 & $63(54,8)$ & $52(45,2)$ & \\
$\quad$ Não & 198 & $91(46,0)$ & $107(54,0)$ & \\
\hline Reumatismo & & & & 0,021 \\
$\quad$ Sim & 48 & $31(64,6)$ & $17(35,4)$ & \\
$\quad$ Não & 265 & $123(46,4)$ & $142(53,6)$ & \\
\hline Cognição preser- & & & & 0,288 \\
vada & & & & \\
$\quad$ Sim & 260 & $124(47,7)$ & $136(52,3)$ & \\
$\quad$ Não & 52 & $29(55,8)$ & $23(44,2)$ & \\
\hline ABVDs & & & & 0,000 \\
$\quad$ Independente & 290 & $134(46,2)$ & $156(53,8)$ & \\
$\quad$ Dependente & 23 & $20(87,0)$ & $3(13,0)$ & \\
\hline
\end{tabular}

Referente à associação das condições de saúde e uso de MPI ressaltaram-se associações significativas quanto à dor, quedas, ao uso de álcool, atividade física, diabetes, obesidade, a problemas no sono, ao nervosismo, a problemas cardíacos, depressão, osteoporose, a problemas circulatórios, a reumatismo e ABVD $(p<0,05)$.

Referente à associação entre o uso de MPI e dor, percebe-se que daqueles que sentem dor, $67,5 \%$ fazem uso de MPI. Em relação à prática de atividade física, notase que dos idosos que praticam alguma atividade, somente $37,7 \%$ fazem uso de MPI.

Entre as variáveis dificuldade visual, tabagismo, hipertensão, Alzheimer, problemas de coluna e cognição e o uso de MPI não houve associações significativas. 


\section{DISCUSSÃO}

Neste estudo, observou-se elevada prevalência quanto ao uso de MPI, sendo que quase metade dos idosos entrevistados fazia uso de alguma medicação potencialmente inapropriada. Estudos populacionais nesta temática encontraram prevalência de 9,5\% até 59,2\%, seguindo Beers 2012 $2^{17,18,20-22}$. Pesquisa que avaliou mil idosos, atendidos pelo Sistema Único de Saúde e não institucionalizados, encontrou prevalência de $48 \%$ de MPI seguindo Beers 2003, e de 59,2\% seguindo Beers 2012, evento que pode ser explicado pelo fato de que na última atualização do critério, foram incluídos medicamentos com alto uso no Brasil ${ }^{21}$. Em estudo realizado na Espanha com idosos na comunidade, foi encontrado uso de MPI em $44 \%$ dos $\operatorname{casos}^{23}$, pesquisa realizada na Nova Zelândia identificou MPI em 42,7\% dos idosos em comunidade ${ }^{24}$, na Suíça a porcentagem foi de $30,7 \%{ }^{25}$. Seguindo estes achados, estudo realizado, em idosos brasileiros atendidos em ambulatórios, encontrou 26,9\% de prevalência quanto ao uso de MPI, podendo perceber que as ocorrências se assemelham em diferentes cenários ${ }^{26}$.

Neste estudo um terço dos idosos fazia uso de cinco ou mais medicamentos, caracterizando polifarmácia $^{11,27-29}$. Já, o estudo de Galato, Silva, Tibúrcio, ${ }^{27}$ com idosos residentes na cidade no sul de Santa Catarina, evidenciou uso de 3,5 medicamentos em média por idoso. Esta condição está associada ao fato de os idosos serem portadores de duas ou mais doenças crônicas, como hipertensão, diabetes, doenças cardíacas e osteomusculares, sendo que quanto maior o número de patologias, mais elevada as prescrições medicamentosas, demonstrando que a terapêutica aplicada ao idoso é quase que totalmente por meio de tratamento farmacológico ${ }^{1,13}$.

Embora a variável sexo não tenha se apresentado significativamente associada ao uso de MPI, chama atenção o fato de o número de mulheres que utilizavam MPI ser quase duas vezes maior que o número de homens, corroborando com o encontrado na literatura. Tal evidência sugere que a maior frequência quanto ao uso de MPI pode estar relacionada ao fato de as mulheres procurarem mais os serviços de saúde, receberem diagnóstico médico, e, consequentemente, se exporem mais aos fármacos inapropriados ${ }^{18,20,21,26}$. Estudo realizado no Canadá com idosos na comunidade identificou que as mulheres receberam mais MPI que os homens, o que pode ser atribuído à prescrição de benzodiazepínicos, antidepressivos e AINES, ocasionadas por questões biológicas, fatores clínicos e socioeconômicos ${ }^{30}$.

Há mais de uma década, diversas pesquisas populacionais tratam do uso de medicamentos em idosos urbanos, utilizando diferentes métodos de avaliação, nos mais diferentes cenários ${ }^{22,26,31}$, inclusive, no âmbito internacional, há registros de estudos com enfoque rural ${ }^{32}$. No Brasil, são poucos os estudos de base populacional que abordam a farmacoterapia em idosos na comunidade, utilizando o critério de Beers, versão 2012, para avaliar os MPI $^{33}$. Nesse estudo, no que se refere à zona de moradia, a maioria dos idosos da área rural fazia uso de MPI, diferentemente dos resultados encontrados no estudo realizados no interior de Minas Gerais, o qual não apresentou associação entre o uso de MPI e zona de moradia $^{20}$.

Quanto à percepção de saúde nos idosos, a autoavaliação foi mais positiva naqueles que não utilizavam MPI. Estudo que aborda fatores associados à percepção negativa de saúde em idosos ${ }^{9}$, reforça que o uso regular de medicamentos favorece uma imagem menos estimada de si, contribuindo para uma avaliação negativa da saúde. Corroborando com esta ideia, estudo que verifica fatores relacionados ao uso dos serviços de saúde por idosos, refere que quanto pior a percepção de saúde mais determinante será o uso de serviços de saúde, o que colabora na indicação de medicações, no uso de MPI $^{34}$ e na autopercepção negativa de saúde ${ }^{20}$.

A maioria dos idosos entrevistados referiu sentir algum tipo de dor, destes $67,5 \%$ utilizavam algum tipo de MPI. A ocorrência da dor eleva o risco de utilizar MPI, já que grande parte dos idosos pratica a automedicação, seja pela propaganda massiva de fármacos, dificuldade de acesso a serviços de saúde ou mesmo a facilidade na aquisição Visto que em nosso país é possível adquirir medicamentos sem prescrição médica, AINES e relaxantes musculares são os fármacos de primeira escolha $\mathrm{e}$ amplamente comercializados, o que agrava o problema de exposição dos idosos aos MPI. Nesse estudo, entre os idosos que relataram problemas de coluna e reumatismo, doenças que constantemente causam dor, a maioria fazia 
uso de MPI. Pesquisa de Manso, Biffi, Gerardi ${ }^{19}$ demonstrou que idosos de comunidade, atendidos por um plano de saúde de São Paulo, tinham como principais MPI prescritos os fármacos da categoria AINES. Esses fármacos devem ser utilizados com máxima cautela, levando em consideração a necessidade de segurança cardiovascular, gastrintestinal, cerebrovasculares e renais, sobretudo nos idosos, não assegurada nestes medicamentos ${ }^{35}$.

Pesquisas evidenciam que os benzodiazepínicos são alguns dos fármacos mais utilizados pelos idosos, nos mais diferentes cenários, o que também foi confirmado nesse estudo $0^{9,18,26,33,36}$. Contrariando esses achados, estudo que avaliou o uso de benzodiazepínicos em idosos na comunidade ${ }^{37}$ apontou prevalência de $6,5 \%$ de uso nas últimas duas semanas, muito abaixo do encontrado em países desenvolvidos. Vale destacar que o uso desses fármacos pode estar ligado à predisposição às quedas. Conforme estudo de revisão sistemática ${ }^{38}$, o uso de benzodiazepínicos representa potencial fator de risco para quedas em idosos, devido a possibilidade de indução de sedação, comprometendo função motora e psíquica. Ain$\mathrm{da}$, os benzodiazepínicos não são recomendados como fármaco de primeira escolha no tratamento de idosos, sobretudo por longos períodos ${ }^{39}$ Outras pesquisas também confirmam esta posiçãa ${ }^{17,20}$. No presente estudo, dos idosos que relataram terem sofrido alguma queda no último ano, 64,6\% faziam uso de MPI. A minoria dos idosos referiu ter problemas no sono, porém, destes, $86,4 \%$ fazia uso de MPI.

Dos idosos que relataram problemas cardíacos, 82,3\% faziam uso de MPI, incluídos na categoria cardiovascular, segundo o critério de Beers, versão 2012, uma classe de medicamentos amplamente utilizada pela população idosa ${ }^{8,13,18}$. Estudo brasileiro realizado com idosos atendidos em ambulatório apresentou prevalência de $37,4 \%$ quanto ao uso de drogas cardiovasculares, em grande parte pelos casos de hipertensão ${ }^{40}$. No presente estudo, a hipertensão foi a patologia autorreferida mais prevalente corroborando resultados de outras pesquisas brasileiras ${ }^{19,23,33,41}$.

Referente aos hábitos de vida, percebeu-se que idosos que praticavam atividade física somavam mais da metade da população estudada e consumiam menos MPI comparado àqueles que não praticavam atividade e utilizavam MPI. Ainda, uma minoria dos idosos apresentava dependência para ABVD e destes 87,0\% faziam uso de
MPI. Estudos evidenciam que o uso de um ou mais fármacos tem associação com maior dependência em ABVD em idosos $^{42,43}$. Estudos realizados no contexto brasileiro ${ }^{18,20}$ corroboram com os achados, indicando associação entre o uso de MPI e o comprometimento da capacidade funcional.

\section{CONCLUSÃO}

O uso de medicamentos potencialmente inapropriados é uma realidade vivenciada por quase metade dos idosos estudados, sobretudo, mulheres, sendo os benzodiazepínicos e os anti-inflamatórios não esteroidais os mais utilizados na população estudada. Pode-se ressaltar que o uso de medicamentos potencialmente inapropriados relaciona-se às condições de dor, quedas, uso de álcool, atividade física, diabetes, obesidade, problemas no sono, nervosismo, problemas cardíacos, depressão, osteoporose, problemas circulatórios, reumatismo e atividades básicas da vida diária.

Frente aos achados é imprescindível que haja sensibilização dos profissionais de saúde quanto à necessidade de uma sistemática revisão dos medicamentos prescritos aos idosos na prática clínica. Por outro lado, devem-se investigar mecanismos de avaliação com vistas a minimizar o número de fármacos indicados e, por conseguinte, as complicações decorrentes do uso inadequado. Nesta perspectiva, o Critério de Beers, versão 2012, mostrou-se como uma possibilidade de avaliação dos fármacos inapropriados, contudo esta lista carece de mais estudos, os quais possam ponderar características mais apropriadas à realidade brasileira.

\section{REFERÊNCIAS}

1. Carvalho MHR, Carvalho SMR, Laurenti R, Payão SLM. Tendência de mortalidade de idosos por doenças crônicas no município de Marília-SP, Brasil: 1998 a 2000 e 2005 a 2007. Epidemiol. Serv. Saúde. [internet] 2014 [acesso em 2017 Nov 26]; 23(2): 347-54. Disponível em: https://repositorio.unesp.br/bitstream/ handle/11449/117930/S2237-96222014000100347. pdf?sequence $=1 \&$ isAllowed $=y$ doi: $\mathrm{http}: / / \mathrm{dx}$.doi. org/10.5123/S1679-49742014000200016 
2. Pimenta FB, Pinho L, Silveira MF, Botelho ACC. Fatores associados a doenças crônicas em idosos atendidos pela Estratégia de Saúde da Família. Ciênc. saúde coletiva. [internet] 2015 Ago [acesso em 2017 Jun 24]; 20 (8): 2489-98. Disponível em: http:// www.scielo.br/scielo.php?script $=$ sci_arttext\&pi$\mathrm{d}=$ S1413-81232015000802489\&lng=en. http://dx. doi.org/10.1590/1413-81232015208.11742014.

3. Veras RP. Prevenção de doenças em idosos: os equívocos dos atuais modelos. Cad.Saúde Pública. [internet] 2012 [acesso em 2017 Ago 22]; 28(10): 1834-40. Disponível em: http://www. scielo.br/scielo.php?script $=$ sci_arttext\&amp; pi$\mathrm{d}=$ S0102-311X2012001000003\&amp;lng=en. http:// dx.doi.org/10.1590/S0102-311X2012001000003.

4. Santiago FS. Projeções dos impactos econômicos decorrentes das mudanças demográficas no Brasil para o período de 2010 a 2050. Belo Horizonte: Faculdade de Ciências Econômicas de Minas Gerais, 2014. Tese doutorado. Disponível em: file://C:/Users/Andressa/ Downloads/FLAVIANE\%20SOUZA\%20SANTIAGO.pdf

5. Instituto Brasileiro de Geografia e Estatística. Pesquisa Nacional de Saúde 2013 - Percepção do estado de saúde, estilos de vida e doenças crônicas. Rio de Janeiro [acesso em 2016 set. 27]. Disponível em: http:// www.ibge.gov.br.

6. Vasconcelos AMN, Gomes MMF. Demographic transition: the brazilian experience. Epidemiol. Serv. Saúde. [internet] 2012 Dez [acesso em 2017 Nov 13]; 21(4): 539-48. Disponível em: http://scielo.iec.gov.br/scielo.php?script $=$ sci_arttext\&pi$\mathrm{d}=$ S1679-49742012000400003\&lng=pt. http://dx. doi.org/10.5123/S1679-49742012000400003.

7. Mendes ACG, Sá DA, Miranda GMD, Lyra TM, Tavares RAW. Assistência pública de saúde no contexto da transição demográfica brasileira: exigências atuais e futuras. Cad. Saúde Pública [internet] 2012 May [acesso em 2017 Jul 27]; 28(5): 955-964. Disponível em: http://www.scielo.br/scielo.php?script $=$ sci_arttext\&pid=S0102-311X2012000500014\&lng=en. http:// dx.doi.org/10.1590/S0102-311X2012000500014.
8. Dal Pizzol TS, Pons ES, Hugo FN, Bozzetti MC, Sousa MLR, Hilgert JB. Uso de medicamentos entre idosos residentes em áreas urbanas e rurais de município no Sul do Brasil: um estudo de base populacional. Cad. Saúde Pública. [internet] 2012 Jan [acesso em 2017 Mar 14]; 28(1): 104-14. Disponível em: http://www. scielo.br/pdf/csp/v28n1/11.pdf doi: http://dx.doi. org/10.1590/S0102-311X2012000100011.

9. Silva RJS, Smith-Menezes A, Tribess S, Rómo-Perez V, Virtuoso JJS. Prevalência e fatores associados à percepção negativa da saúde em pessoas idosas no Brasil. Rev. bras. Epidemiol. [internet] 2012 [acesso em 2017 Jun 20]; 15(1): 49-62. Disponível em: http:// www.scielo.br/scielo.php?script $=$ sci_arttext\&pi$\mathrm{d}=$ S1415-790X2012000100005\&lng=en. http://dx. doi.org/10.1590/S1415-790X2012000100005.

10. Azevedo ALS, Silva RA, Tomasi E, Quevedo LA. Doenças crônicas e qualidade de vida na atenção primária à saúde. Cad. Saúde Pública. [internet] 2013 Set [acesso em 2017 Ago 20]; 29(9): 1774-82. Disponível em: http://www.scielo.br/pdf/csp/v29n9/a17v29n9.pdf doi: http://dx.doi.org/10.1590/0102-311X00134812.

11. Carvalho MFC, Romano-Lieber NS, Bergsten-Mendes G, Secoli SR, Ribeiro E, Lebrão ML, et al. Polifarmácia entre idosos do Município de São Paulo - Estudo SABE. Rev. bras. epidemiol. [internet] 2012 Dez [acesso em 2017 Out 14]; 15(4): 817-27. Disponível em: http://www.scielo.br/scielo.php?script $=$ sci_arttext\&pid=S1415-790X2012000400013 doi: http:// dx.doi.org/10.1590/S1415-790X2012000400013.

12.Alomar MJ. Factors affecting the development of Adverse Drug Reactions. Saudi Pharmaceutical Journal. [internet] $2014 \mathrm{Fev}$ [acesso em 2017 Ago 01]; 22(2): 83-94. Disponível em: https://www.ncbi.nlm. nih.gov/pmc/articles/PMC3950535/ doi: 10.1016/j. jsps.2013.02.003.

13. Neves SJF, Marques APO, Leal MCC, Diniz AS, Medeiros TS, Arruda IKG. Epidemiologia do uso de medicamentos entre idosos em área urbana do Nordeste do Brasil. Rev. Saúde Pública. [internet] 2013 Ago [acesso em 2017 Set 13]; 47 (4): 759-68. Disponível em: http://www.scielo.br/scielo.php?script $=$ sci_arttext\&pid=S0034-89102013000400759\&lng=en. http:// 
dx.doi.org/10.1590/S0034-8910.2013047003768.

14. Alves CAD. Alterações farmacológicas na terapêutica geriátrica [dissertação]. Faro: Universidade do Algarve; 2012. 108 p.

15. Gallagher PF, Barry PJ , Ryan C, Hartigan I, O'Mahony D. Inappropriate prescribing in an acutely ill population of elderly patients as determined by Beers criteria. Age Ageing. 2008;37:96-101. Disponível em: https://pdfs.semanticscholar.org/bb66/5d0b1861efaf2feb5ae04cfa9524b5fbe8c3.pdf.

16. Gorzoni ML, Fabbri RMA, Pires SL. Potentially inappropriate medications in elderly. . Rev. Assoc. Med. Bras. [internet] 2012 Ago [acesso em 2017 Ago 21] ;58(4): 442-446. Disponível em: http:// www.scielo.br/scielo.php?script =sci_arttext\&pi$\mathrm{d}=$ S0104-42302012000400014\&lng=en. http://dx. doi.org/10.1590/S0104-42302012000400014.

17. American Geriatrics Society. Beers Criteria Update Expert Panel. American Geriatrics Society updated Beers criteria for potentially inappropriate medication use in older adults. J Am Geriatr Soc. 2012; 60(4): 616-31.

18. Cassoni TCJ, Corona LP, Romano-Liber NS, Secoli SR, Duarte YAO, Lebrão ML. Uso de medicamentos potencialmente inapropriados por idosos do Município de São Paulo, Brasil: Estudo SABE. Cad. Saúde Pública. [internet] 2014 Ago [acesso em 2017 Nov 20]; 30(8): 1708-20. Disponível em: http://www.scielo.br/pdf/csp/ v30n8/0102-311X-csp-30-8-1708.pdf doi:http://dx.doi. org/10.1590/0102-311X00055613.

19. Manso MEG, Biffi ECA, Gerardi TJ. Prescrição inadequada de medicamentos a idosos portadores de doenças crônicas em um plano de saúde no município de São Paulo, Brasil. Rev. bras. geriatr. gerontol. [internet] 2015 Mar [acesso em 2017 Abr 27];18(1): 151-164. Disponível em: http:// www.scielo.br/scielo.php?script =sci_arttext\&pi$\mathrm{d}=$ S1809-98232015000100151\&lng=en. http://dx. doi.org/10.1590/1809-9823.2015.14056.

20. Martins GA, Acurcio FA, Franceschini SCC, Priore SE, Ribeiro AQ. Uso de medicamentos potencialmente inadequados entre idosos do Município de Viçosa,
Minas Gerais, Brasil: um inquérito de base populacional. Cad. Saúde Pública [internet] 2015 Nov [acesso em 2017 Abr 27]; 31(11): 2401-2412. Disponível em: http://www.scielo.br/scielo.php?script $=$ sci_arttext\&pid=S0102-311X2015001102401\&lng=en. http:// dx.doi.org/10.1590/0102-311X00128214

21. Baldoni AO, Ayres LR, Martinez EZ, Dewulf Nde L, Dos Santos V, Pereira LR. Factors associated with potentially inappropriate medications use by the elderly according to Beers criteria 2003 and 2012. Int J Clin Pharm. [internet] 2014 Abr [acesso em 2017 Set 09]; 36(2): 316-24. Disponível em: https://ink.springer.com/article/10.1007\%2Fs11096-013-9880-y doi: 10.1007/s11096-013-9880-y.

22. Santos TRA, Lima DM, Nakatani AYK, Pereira LV, Leal GS, Amaral RG. Medicine use by the elderly in Goiania, Midwestern Brazil. Rev. Saúde Pública. [internet] $2013 \mathrm{Fev}$ [acesso em 2017 Out 18]; 47(1): 94-103. Disponível em: http:// www.scielo.br/scielo.php?script $=$ sci_arttext\&pi$\mathrm{d}=$ S0034-89102013000100013\&lng=en. http://dx. doi.org/10.1590/S0034-89102013000100013.

23. Blanco-Reina E, Ariza-Zafra G, Ocaña-Riola R, León -Ortiz M. 2012 American Geriatrics Society Beers Criteria: enhanced applicability for detecting potentially inappropriate medications in European older adults? a comparison with the Screening Tool of Older Person's potentially inappropriate Prescriptions. Journal of the American Geriatrics Society. [internet] 2014 [acesso em 2017 Dez 12]; 62(7):1217-23. Disponível em: https://www.ncbi.nlm.nih.gov/pubmed/24917083 doi: $10.1111 /$ jgs. 12891.

24. Nishtala PS, Bagge ML, Campbell AJ, Tordoff JM. Potentially inappropriate medicines in a cohort of community-dwelling older people in New Zealand. Geriatrics \& gerontologyinternational. 2014; 14 (1): 89-93. Disponível em: http://onlinelibrary.wiley.com/ doi/10.1111/ggi.12059/

25. Blozik E, Rapold R, Reich O. Prescription of potentially inappropriate medication in older persons in Switzerland: does the dispensing channel make a 
difference? Risk Mang Healthc Policy. [internet] 2015 [acesso em 2017 Mar 23]; 24(8):73-80. Disponível em: https://www.ncbi.nlm.nih.gov/pubmed/25977609 doi: 10.2147/RMHP.S78179.

26. Faustino CG, Passarelli MCG, Jacob-Filho W. Potentially inappropriate medications among elderly Brazilian outpatients. Med. J. [internet] 2013 [acesso em 2017 Mar 22]; 131(1): 19-26. Disponível em: http:// www.scielo.br/scielo.php?script $=$ sci_arttext\&pi$\mathrm{d}=$ S1516-31802013000100019\&lng=en. http://dx. doi.org/10.1590/S1516-31802013000100004.

27. Galato D, Silva ES, Tiburcio LS. Estudo de utilização de medicamentos em idosos residentes em uma cidade do sul de Santa Catarina (Brasil): um olhar sobre a polimedicação. Ciênc. saúde coletiva [internet] 2010 Set [acesso em 2017 Fev 21]; 15(6): 2899-2905. Disponível em: http:// www.scielo.br/scielo.php?script $=$ sci_arttext\&pi$\mathrm{d}=$ S1413-81232010000600027\&lng=en. http://dx. doi.org/10.1590/S1413-81232010000600027.

28. Heuberger R. Polypharmacy and food-drug interactions among older persons: a review. Jou Nut Gerontol Geriatr. [internet] $2012 \mathrm{Nov}$ [acesso em Ago 22];31(4): 325-403. Disponível em: https://www.tandfonline.com/doi/abs/10.1080/21551197.2012.729902.

29. Secoli SR. Polifarmácia: interações e reações adversas no uso de medicamentos por idosos. Rev. bras. enferm. [internet] 2010 [acesso em 2017 Mar 14]; 63(1): 136-40. Disponível em: http:// www.scielo.br/scielo.php?script $=$ sci_arttext\&pi$\mathrm{d}=$ S0034-71672010000100023\&lng=en. http://dx. doi.org/10.1590/S0034-71672010000100023.

30. Morgan SG, Weymann D, Pratt B, Smolina K, Gladstone EJ, Raymond C, et al. Sex differences in the risk of receiving potentially inappropriate prescriptions among older adults. Age Ageing. [internet] $2016 \mathrm{Jul}$ [acesso em 2017 Ago 20]; 45 (4): 535-42. Disponível em: https://www.ncbi.nlm.nih.gov/pmc/articles/ PMC4916346/ https://academic.oup.com/ageing/article/45/4/535/1681337
31. Sousa-Muñoz RL, Ibiapina GR, Gadelha CS, Maroja JLS. Prescrições geriátricas inapropriadas e polifarmacoterapia em enfermarias de clínica médica de um Hospital-Escola. Rev. bras. geriatr. Gerontol. [internet] 2012 [acesso em 2017 Fev 15]; 15(2):315-24. Disponível em: http://www.scielo.br/scielo.php?script $=$ sci_arttext\&pid=S1809-98232012000200014\&lng=en. http:// dx.doi.org/10.1590/S1809-98232012000200014.

32.Grymonpre RE, Hawranik PG. Rural residence and prescription medication use by community-dwelling older adults: a review of the literature. Joul Rural Health. [internet] $2008 \mathrm{Mar}$ [acesso em 2017 Set 16];24: 203-9. Disponível em: http://onlinelibrary. wiley.com/doi/10.1111/j.1748-0361.2008.00159.

33.Lopes LM, Figueiredo TP, Costa SC, R AMM. Utilização de medicamentos potencialmente inapropriados por idosos em domicílio. Ciênc. saúde coletiva [internet] 2016 Nov [acesso em 2017 Mai 10]; 21(11): 3429-3438. Disponível em: http:// www.scielo.br/scielo.php?script $=$ sci_arttext\&pi$\mathrm{d}=$ S1413-81232016001103429\&lng=en. http://dx. doi.org/10.1590/1413-812320152111.14302015.

34. Pagotto V, Bachion MM, Silveira EA. Autoavaliação da saúde por idosos brasileiros: revisão sistemá- tica da literatura. Rev Panam Salud Publica. 2013;33(4):30210. Disponível em:https://www.scielosp.org/article/ ssm/content/raw/?resource_ssm_path $=/$ media/assets/rpsp/v33n4/a10v33n4.pdf

35. Scarpignato C, Lanas A, Blandizzi C, Lems WF, Hermann M, Hunt RH. Safe prescribing of non-steroidal anti-inflammatory drugs in patients with osteoarthritis-an expert consensus addressing benefits as well as gastrointestinal and cardiovascular risks. BMC medicine. [internet] 2015 [acesso em 2017 Out 29]; 13 (1): 55. Disponível em: https://bmcmedicine.biomedcentral.com/articles/10.1186/s12916-015-0285-8 https:// doi.org/10.1186/s12916-015-0285-8

36. Brunoni AR, Nunes MA, Figueiredo R, Barreto SM, da Fonseca Mde J, Lotufo PA, et al. Patterns of benzodiazepine and antidepressant use among middle-aged adults - the Brazilian longitudinal study of adult 
health (ELSA-Brasil). J Affect Disord. [internet] 2013 Out [2017 Jun 16]; 151(1):71-7. Disponível em: https://www.ncbi.nlm.nih.gov/pubmed/23769607 doi: 10.1016/j.jad.2013.05.054.

37. Cunha CDA, Souza MCC, Cattanio GAA, Iahnn SR, Lima RC. Benzodiazepine use and associated factors in elderly in the city of Dourados, MS, Brazil. J. bras. psiquiatr.[internet] 2015 Set [acesso em $2017 \mathrm{Abr}$ 21]; 64(3): 207-12. Disponível em: http://www.scielo. br/pdf/jbpsiq/v64n3/0047-2085-jbpsiq-64-3-0207.pdf doi: http://dx.doi.org/10.1590/0047-2085000000080.

38. Rezende CP, Gaede-Carrillo MRG, Sebastião ECO. Queda entre idosos no Brasil e sua relação com o uso de medicamentos: revisão sistemática. Cad Saúde Pública. 2012; 28(12): 2223-35. Disponível em: http:// www.scielo.br/pdf/csp/v28n12/02.pdf

39. Lader M. Benzodiazepine harm: how can it be reduced? Br. J. ClinPharmacolv. [internet] 2014 Fev [acesso em Maio 10];77(2) 295-301. Disponível em: $\quad$ http://onlinelibrary.wiley.com/doi/10.1111 /j.1365-2125.2012.04418.

40. Cuentro VS, Andrade MA, Gerlack LF, Bós ÂJG, Silva MVS, Oliveira AF. Prescrições medicamentosas de pacientes atendidos no ambulatório de geriatria de um hospital universitário: estudo transversal descritivo. Ciência \& Saúde Coletiva. [internet] 2014 [acesso em 2017 Ago 24]; 19(8): 3355-64. Disponível em: http://www.scielo.br/pdf/csc/v19n8/14138123-csc-19-08-03355.pdf doi: http://dx.doi. org/10.1590/1413-81232014198.09962013.

41. Morelato RL, Pacheco EO, Laghi CR, Juri LS, Faé IS. Frequência de prescrição de fármacos considerados de uso potencialmente inapropriado em pessoas idosas. Salus J Health SCi [internet] 2015 [acesso em 2017 Set]; 1(1): 27-36. Disponível em: file://C:/Users/ Lu\%C3\%ADs\%20Henrique/Downloads/Portugu\%C3\%AAs6.pdf. doi: https://dx.doi.org/10.5935/24477826.20150004.

42. Brito TA, Fernandes MH, Coqueiro RS, Jesus CS, Freitas R. Functional capacity and associated factors among longevous senior individuals living in community: a population study in Northeastern Brazil. Fisioter. Pesqui. [internet] 2014 [acesso em 2017 Jun 16]; 21(4): 308-13. Disponível em: http://www.scielo.br/scielo. php? pid $=S 1809=29502014000400308-\&$ script sci_abstract doi: http://dx.doi.org/10.590/18092950/11556021042014.

43. Freitas RS, Fernandes MH, Coqueiro RS, Reis Júnior WM, Rocha SV, Brito TA. Capacidade funcional e fatores associados em idosos: estudo populacional. Acta paul. enferm. [internet] 2012 [acesso em 2017 Jul 15]; 25(6): 933-939. Disponivel em: http:// www.scielo.br/scielo.php?script $=$ sci_arttext\&pi$\mathrm{d}=$ S0103-21002012000600017\&lng=en. http://dx. doi.org/10.1590/S0103-21002012000600017.

Recebido em: 29/08/2017 Aceito em: 01/03/2018 